Rev Biomed 2001; 12:19-25.

\title{
Frecuencia de parásitos gastrointestinales en animales domésticos diagnosticados en Yucatán, México.
}

\section{Artículo Original}

Roger I. Rodríguez-Vivas, Ligia A. Cob-Galera, José L. Domínguez-Alpizar.

Universidad Autónoma de Yucatán. Facultad de Medicina Veterinaria y Zootecnia. Departamento de Parasitología. Mérida, Yucatán, México.

\section{RESUMEN.}

Introducción. Los parásitos gastrointestinales ocasionan grandes pérdidas a la producción y salud animal. La información generada en los laboratorios de diagnóstico ayudan en el conocimiento de las parasitosis y permiten diseñar programas de prevención, control y/o erradicación. Material y métodos. Se revisaron los archivos del laboratorio de Parasitología de la Facultad de Medicina Veterinaria y Zootecnia de la Universidad Autónoma de Yucatán, de marzo de 1984 a diciembre de 1999. Se obtuvo la información de las muestras de heces de animales domésticos que fueron remitidas y procesadas mediante la técnica de Flotación Centrifugada.

Resultados. Se analizaron un total de 10689 muestras fecales, de las cuales 3827 fueron de bovinos, 1456 de caprinos, 544 de ovinos, 993 de caninos, 46 de felinos, 211 de aves, 3232 de porcinos y 380 de equinos. Los parásitos gastrointestinales más frecuentes en las distintas especies animales fueron los siguientes: bovinos: strongylida $(60.64 \%)$ y coccidia $(71.57 \%)$, cabras: strongylida (75.41\%) y coccidia $(93.40 \%)$, ovinos: strongylida $(59.00 \%)$ y coccidia $(91.17 \%)$, caninos: Ancylostoma sp (37.36\%), felinos: Ancylostoma sp (32.61\%), aves de corral: coccidia (53.00\%), porcinos: coccidia (45.04\%), y equinos: Strongylus sp $(55.26 \%)$.

Conclusión. Los animales domésticos del estado de Yucatán, México, se encuentran parasitados por una gran variedad de nemátodos, céstodos y protozoarios.

(Rev Biomed 2001; 12:19-25)

Palabras clave: Parásitos gastrointestinales,

Solicitud de sobretiros: MSc. Roger I. Rodríguez-Vivas, Depto. de Parasitología, Fac. de Medicina Veterinaria y Zootecnia, Universidad Autónoma de Yucatán, Km. 15.5 Carr. Mérida-Xmatkuil, Apdo. Postal 4-116 Itzimná, C.P. 97100, Mérida, Yucatán, México. Tel. (9) 942-32-00 Fax: (9) 942-32-05 E-mail: rvivas@tunku.uady.mx

Recibido el 24/Marzo/2000. Aceptado para publicación el 7/Julio/2000. 
RI Rodríguez-Vivas, LA Cob-Galera, JL Domínguez-Alpizar.

animales domésticos, strongylida, coccidia, Ancylostoma, Strongylus, Yucatán México.

\section{SUMMARY.}

Frequency of gastrointestinal parasites in domestic animals, diagnosed in Yucatan, Mexico.

Introduction. Gastrointestinal parasites cause damage to animal health and production. The laboratory information is a tool in the knowledge of parasitisis. This information allows for the design of preventive, control and eradication programs.

Material and methods. Data from the Parasitology Laboratory of the Facultad de Medicina Veterinaria y Zootecnia of the Universidad Autonoma de Yucatan, from March 1984 to December 1999, were analyzed. Information of fecal samples from the most important domestic animals were obtained and tested by the centrifuge flotation technique.

Results. A total of 10689 faecal samples were analyzed. The fecal samples were from the following animal species: bovine 3827, goats 1456 , sheep 544, canine 993 , feline 46 , domestic fowl 211, swine 3232 and equine 380 . The more frequent gastrointestinal parasites in different animal species were: bovine: strongylida $(60.64 \%)$ and coccidia $(71.57 \%)$, goats: strongylida (75.41\%) and coccidia (93.40\%), sheep: strongylida (59.00\%) and coccidia (91.17\%), canine: Ancylostoma sp (37.36\%), feline: Ancylostoma sp (32.61\%), domestic fowl: coccidia (53.00\%), swine: coccidia (45.04\%), and equine: Strongylus sp (55.26\%).

Conclusion. It is concluded that the domestic animals from the state of Yucatan, are parasitized by a wide range of nematodes, cestodes and protozoa. (Rev Biomed 2001; 12:19-25)

Key words: gastrointestinal parasites, domestic animals, strongylida, coccidia, Ancylostoma, Strongylus, Yucatan Mexico.

\section{Revista Biomédica}

\section{INTRODUCCION.}

Los animales domésticos se encuentran expuestos a numerosos microorganismos tales como bacterias, virus, rickettsias, mycoplasmas, clamidias, hongos y parásitos. Las parasitosis gastrointestinales son generalmente producidas por helmintos (nemátodos, céstodos) y protozoarios. Estos representan una amenaza para los animales domésticos, ya que causan anorexia, reducción en la ingestión de alimentos, pérdidas de sangre y proteínas plasmáticas en el tracto gastrointestinal, alteraciones en el metabolismo proteíco, reducción de minerales, depresión en la actividad de algunas enzimas intestinales y diarrea $(1,2)$. En los animales productivos los parásitos gastrointestinales (PGI) reducen la producción de carne, leche, huevo, lana y otros productos para el consumo y uso humano; en los animales de deporte reducen el rendimiento físico y en los animales de compañía representan un importante riesgo de transmisión de parásitos a los humanos (3).

La información generada en las investigaciones, hallazgos clínicos de campo, hallazgos en rastros y reportes de clínicas y laboratorios, es de suma importancia en el diagnóstico de situación de las principales enfermedades en los animales domésticos (4). Esta información permite tener elementos para sentar las bases para el diseño de programas de prevención, control y erradicación de las enfermedades en diferentes regiones (5).

El objetivo del presente trabajo es proporcionar información sobre la frecuencia de los PGI diagnosticados en el laboratorio de Parasitología de la Facultad de Medicina Veterinaria y Zootecnia de la Universidad Autónoma de Yucatán (FMVZ-UADY), mediante exámenes de heces en los principales animales domésticos.

\section{MATERIAL Y METODOS.}

El clima del Estado de Yucatán es tropical húmedo con lluvias en verano. La temperatura 
Parásitos gastrointestinales en animales domésticos.

promedio anual fluctúa entre los $24.6 \mathrm{C}$ y $27.7 \mathrm{C} \mathrm{y}$ la humedad relativa llega a $80 \%$ en la costa y $72 \%$ en el interior. La precipitación pluvial anual oscila entre $415 \mathrm{~mm}$ y $1290 \mathrm{~mm}(6)$.

El Laboratorio de Parasitología de la FMVZUADY, se encuentra localizado en el municipio de Mérida, Yucatán, México.

Para hacer el reporte de los PGI diagnosticados en los principales animales domésticos, se revisaron los archivos del laboratorio de Parasitología de la FMVZ-UADY, de marzo de 1984 a diciembre de 1999. Se obtuvo la información de las muestras de heces que fueron remitidas al laboratorio y procesadas mediante la técnica de Flotación Centrifugada descrita por Rodríguez y col. (7). Se registraron las siguientes variables: procedencia (Estado de Yucatán y otros Estados), especie animal (bovino, caprino, ovino, porcino, canino, felino, ave y equino) y tipo de parásito (orden, género y/o especie). Las muestras remitidas al laboratorio provenían de animales clínicamente sanos y enfermos, y de distinta raza, especie, edad, condición corporal y procedencia. Con esta información se elaboró una base de datos mediante el programa PANACEA (PAN Livestock Service, Reading University, U.K., Versión 1989) y se obtuvieron las frecuencias de los parásitos en relación a la especie animal.

\section{RESULTADOS.}

En el período de estudio se analizaron un total de 10689 muestras de heces de animales domésticos, de las cuales 3827 fueron de bovinos, 1456 de caprinos, 544 de ovinos, 993 de caninos, 46 de felinos, 211 de aves, 3232 de porcinos y 380 de equinos.

En el cuadro 1 se presenta la frecuencia de casos positivos a PGI diagnosticados en heces de bovinos, caprinos y ovinos. Se observa que en las tres especies animales el orden coccidia es el más frecuente con $71.57 \%, 93.40 \%$ y $91.17 \%$ respectivamente.

En el cuadro 2 se presenta la frecuencia de casos positivos a PGI diagnosticados en heces de caninos y felinos. Se observa que en ambas especies animales el género Ancylostoma es el más frecuente con $37.36 \%$ y $32.60 \%$ respectivamente.

En el cuadro 3 se presenta la frecuencia de casos positivos a PGI diagnosticados en heces de porcinos. Se observa que el orden coccidia es el más frecuente con $45.04 \%$.

En 211 heces de aves de corral procesadas, se encontraron tres géneros y un orden. La frecuencia encontrada fue: Ascaridia sp (15.16), Heterakis sp (15.17\%), Capilaria sp (6.63\%) y coccidia $(53.08 \%)$.

En 380 heces de equinos procesadas, se

Cuadro 1

Parásitos gastrointestinales diagnosticados en heces de bovinos, caprinos y ovinos examinadas en el Laboratorio de Parasitología de la FMVZ-UADY* de marzo de 1984 a diciembre de 1999.

\begin{tabular}{|c|c|c|c|c|c|c|}
\hline \multirow[t]{2}{*}{ PARASITO } & \multicolumn{2}{|c|}{$\begin{array}{c}\text { BOVINOS } \\
(\mathbf{n}=3827)\end{array}$} & \multicolumn{2}{|c|}{$\begin{array}{c}\text { CAPRINOS } \\
(n=1456)\end{array}$} & \multicolumn{2}{|c|}{$\begin{array}{c}\text { OVINOS } \\
(n=544)\end{array}$} \\
\hline & $N^{0}$ de heces & $(\%)$ & $N^{0}$ de heces & $(\%)$ & $N^{0}$ de heces & $(\%)$ \\
\hline Strongylida $\uparrow$ & 2321 & (60.64) & 1098 & $(75.41)$ & 321 & (59.00) \\
\hline Strongyloides $\mathrm{sp}$ & 378 & $(9.87)$ & 455 & $(31.25)$ & 127 & $(23.34)$ \\
\hline Trichuris sp & 317 & $(8.28)$ & 223 & $(15.31)$ & 175 & $(32.16)$ \\
\hline Toxocara vitulorum & 8 & $(0.20)$ & 0 & & 0 & \\
\hline Capillaria $\mathrm{sp}$ & 29 & $(0.75)$ & 0 & & 0 & \\
\hline Moniezia sp & 148 & $(3.86)$ & 16 & (1.09) & 86 & $(15.80)$ \\
\hline coccidia* $^{*}$ & 2739 & (71.57) & 1360 & $(93.40)$ & 496 & (91.17) \\
\hline
\end{tabular}

* FMVZ-UADY = Facultad de Medicina Veterinaria y Zootecnia de la Universidad Autónoma de Yucatán.

$\dagger$ Se reporta como orden de parásito. 
RI Rodríguez-Vivas, LA Cob-Galera, JL Domínguez-Alpizar.

Cuadro 2

Parásitos gastrointestinales diagnosticados en heces de caninos y felinos examinadas en el laboratorio de

Parasitología de la FMVZ-UADY* de marzo de 1984 a diciembre de 1999.

\begin{tabular}{lrlccc}
\hline PARÁSITO & \multicolumn{2}{c}{$\begin{array}{c}\text { CANINOS } \\
(\mathbf{n = 9 9 3})\end{array}$} & \multicolumn{2}{c}{$\begin{array}{c}\text { FELINOS } \\
(\mathbf{n}=\mathbf{4 6})\end{array}$} \\
& $\mathbf{N}^{\mathbf{0}}$ de heces & $(\boldsymbol{\%})$ & $\mathbf{N}^{\mathbf{0}}$ de heces & $(\boldsymbol{\%})$ \\
\hline Capillaria sp & 16 & $(1.61)$ & 3 & $(6.52)$ \\
Toxocara sp & 77 & $(7.75)$ & 2 & $(4.34)$ \\
Ancylostoma sp & 371 & $(37.36)$ & 15 & $(32.60)$ \\
Toxascaris leonina & 15 & $(1.51)$ & 10 & $(21.73)$ \\
Dipylidium caninum & 7 & $(0.70)$ & 8 & $(17.39)$ \\
Trichuris vulpis & 73 & $(7.35)$ & 0 & \\
Coccidia $\dagger$ & 95 & $(9.56)$ & 7 & $(15.21)$ \\
\hline
\end{tabular}

* FMVZ-UADY = Facultad de Medicina Veterinaria y Zootecnia de la Universidad Autónoma de Yucatán.

$\dagger$ Se reporta como orden de parásito.

\section{Cuadro 3}

Parásitos gastrointestinales diagnosticados en heces de porcinos $(n=3232)$ examinadas en el laboratorio de Parasitología de la FMVZ-UADY de marzo de 1984 a diciembre de 1999.

\begin{tabular}{lrl}
\hline Parásito & $\mathbf{N}^{\mathbf{0}}$ de heces & $\mathbf{( \% )}$ \\
\hline Strongyloides $s p$ & 256 & $(7.92)$ \\
Coccidia* & 1456 & $(45.04)$ \\
Ascaris $\mathrm{sp}$ & 257 & $(7.95)$ \\
Oesophagostomum $s p$ & 481 & $(14.88)$ \\
Capillaria $\mathrm{sp}$ & 1 & $(0.03)$ \\
Trichuris $\mathrm{sp}$ & 474 & $(14.66)$ \\
\hline
\end{tabular}

* Se reporta como orden de parásito.

encontraron dos géneros. La frecuencia encontrada fue: Strongylus (55.26) y Parascaris equorum $(15.17 \%)$.

De las 10689 muestras procesadas, 10206 $(95.48 \%)$ provinieron de animales que habitaban en el estado de Yucatán, y 483 (4.52\%) procedieron de otros estados de la República Mexicana.

\section{DISCUSION.}

Las parasitosis gastrointestinales en rumiantes son una de las enfermedades más importantes en las ganaderías tropicales (8-10), ya que reducen la ganancia de peso y producen alta morbilidad y mortalidad en animales jóvenes (1). En el presente trabajo se encontró que los parásitos de los órdenes strongylida y coccidia son los más frecuentes en rumiantes. Estos resultados coinciden con los hallazgos obtenidos en investigaciones realizadas en bovinos $(8,10)$, ovinos $(11)$ y caprinos $(9,12)$ bajo condiciones del estado de Yucatán, México.

Dentro de los parásitos del orden strongylida que afectan a los rumiantes, el género Haemonchus ha sido reportado con mayor frecuencia $(8,9,12)$. Su característica principal es causar un estado de anemia, ya que tanto las larvas de cuarto estadío como los adultos son hematófagos y se calcula que en un animal la pérdida media de sangre es de 0.05 ml por parásito por día (1).

En el estado de Yucatán se encontró que las especies del orden coccidia más importantes en bovinos son Eimeria bovis, E. zuernii, E. ellipsoidalis y $E$. auburnensis (10) y en caprinos E. ninakohlyakimovae, E. caprina, E. arlongi y E. allijevi (9).

A pesar que los órdenes strongylida y coccidia son los más frecuentes en rumiantes, los géneros Strongyloides, Trichuris, Capillaria, Toxocara y Moniezia, pueden producir patologías en los rumiantes cuando se presentan en parasitosis mixtas (1).

El género Ancylostoma fue el más frecuente en las muestras remitidas de caninos $(37.36 \%)$ y felinos $(32.60 \%)$. Este género ha sido reportado como el parásito más frecuente en perros en otras partes del mundo $(13,14)$ y en Yucatán, México (15). El parásito adulto de este género vive desde pocos meses hasta menos de 2 años y es probablemente el parásito más importante de los perros y gatos, ya que es un hematófago ávido, que consume aproximadamente $0.1 \mathrm{ml}$ de sangre al día, además de las hemorragias que ocasiona (16).

Los géneros Ancylostoma, Toxocara y Dipylidium pueden ser agentes de enfermedades

\section{Revista Biomédica}




\section{Parásitos gastrointestinales en animales domésticos.}

zoonóticas $(3,17,18)$. Ancylostoma caninum puede invadir al humano, pudiendo incluso llegar a sus estadíos adultos; sin embargo, el principal efecto patógeno se produce cuando invade al humano por vía percutánea, entidad que recibe el nombre de larva migrans cutánea (3). La larva migrans visceral debida a las larvas de Toxocara canis es un peligro en los niños que ingieren objetos contaminados (19). Rodríguez y col. (20) reportaron que Dipylidium caninum tiene una prevalencia del $18.7 \%$ diagnosticado en heces y $52.0 \%$ diagnosticado en necropsia de perros callejeros de la ciudad de Mérida, Yucatán. Estos valores son superiores a los encontrados en el presente estudio $(0.70 \%)$, pudiendo los resultados estar influenciados por la procedencia de las muestras remitidas para el diagnóstico, ya que en el laboratorio llegan muestras de animales con dueño donde las condiciones sanitarias son mejores. Dipylidium caninum ha sido reportado en infantes que están en estrecho contacto con perros (21-23). Se necesitan estudios en el estado de Yucatán para conocer el papel de los perros y gatos en la transmisión de estos géneros a los humanos.

En aves de corral la coccidiosis es la enfermedad parasitaria más importante, ya que provoca grandes pérdidas económicas debido a la alta mortalidad, retraso en el crecimiento, incremento en la conversión alimenticia y deterioro en la pigmentación de las aves (4). En el estado de Yucatán, Rodríguez y col. (24) determinaron que la coccidiosis es la principal causa de mortalidad en aves de traspatio. La presentación clínica de la coccidiosis depende de ciertos factores tales como la edad, la raza, tipo de explotación y manejo de los animales. Los casos que fueron remitidos al laboratorio provinieron de aves de corral que presentaban diferentes características individuales y de manejo. Los nemátodos de los géneros Heterakis, Ascaridia y Capillaria se presentan en parvadas que se mantienen en pisos, con camas húmedas o con sistemas de bebederos defectuosos que permiten el derramamiento de agua. Cuando se realiza la cría de aves de corral en jaulas, se reducen los problemas con estas parasitosis (2).

Los parásitos entéricos más frecuentes en cerdos fueron los géneros Oesophagostomum y Trichuris, y el orden coccidia. Muirhead (25), Thornton (26), Mackinnon (27) y Cordero y col (28) reportan que las parasitosis pueden llegar a ser de considerable riesgo para la salud de las piaras, si no se establece un programa sanitario adecuado. Vado (29) encontró en cerdos explotados en sistemas extensivos que los géneros Oesophagostomum y Trichuris y el orden coccidia son los parásitos internos más frecuentes. Murrell (30) reporta que los gusanos nodulares (Oesophagostomum $\mathrm{sp}$ ) son los más frecuentes en marranas, especialmente aquellas manejadas en condiciones de pastoreo, llegando a alcanzar prevalencia entre 30 y $50 \%$.

En equinos el género Strongylus produce importantes daños, que varían de acuerdo con la migración que realizan las diferentes especies durante su fase larvaria, así como si los adultos se alimentan de sangre y mucosa o únicamente de contenido intestinal. La larva de S. vulgaris posee un alto grado de patogenicidad $(16,31)$. Cuando penetra en la pared intestinal ejerce acción traumática, apareciendo pequeños puntos hemorrágicos en su trayecto. La frecuencia de este género es muy variable (2). En un estudio realizado en equinos sacrificados en la ciudad de México, Alcázar (32) señala que el $85 \%$ de los animales presentaron huevecillos de Strongylus sp. Pinzón (33) reportó que el 78\% de los equinos de la escuela militar de especialistas en equitación presentaron Strongylus sp. Estos hallazgos son superiores al encontrado en el presente reporte. Se necesitan estudios más profundos en nuestra zona para conocer el impacto del género Strongylus en la industria equina.

El reporte de la frecuencia de estos PGI en el estado de Yucatán (95.48\% de las muestras analizadas procedían del estado de Yucatán) pone de manifiesto la presencia de una gran diversidad de parásitos en los principales animales domésticos. Se hace necesario estudios más profundos 


\section{RI Rodríguez-Vivas, LA Cob-Galera, JL Domínguez-Alpizar.}

encaminados a conocer la importancia de estos PGI en los animales y el hombre.

Se concluye que los animales domésticos del estado de Yucatán, México, se encuentran parasitados por una gran variedad de nemátodos, céstodos y protozoarios.

\section{REFERENCIAS.}

1.- Soulsby EJL. Parasitología y enfermedades parasitarias en los animales domésticos. 7a ed. México: Interamericana; 1987. p. 100-342.

2.- Quiroz RH. Parasitología y enfermedades parasitarias de animales domésticos. México: Limusa; 1989. p. 826.

3.- Acha P, Szyfres B. Zoonosis y enfermedades transmisibles comunes al hombre y a los animales. 2a ed. México: Organización Mundial de la Salud, Organización Panamericana de la Salud; 1988. p. 727-8.

4.- Blood DC, Henderson JA, Radostitis OM. Medicina Veterinaria. México: Interamericana; 1988. p. 1411.

5.- Thrusfield M. Veterinary Epidemiology. $2^{\text {nd }} e d$. Oxford: Blackwell Science; 1995; p. 129-42.

6.- Instituto Nacional de Estadística, Geografía e Informática. Gobierno del Estado de Yucatán. Aspectos geográficos. Anuario estadístico del estado de Yucatán; Yucatán México; 1996. p. 444.

7.- Rodríguez-Vivas RI, Domínguez-Alpizar JL, CobGalera LA. Técnicas parasitológicas en medicina veterinaria. Mérida: Universidad Autónoma de Yucatán; 1994. p. 38-45.

8.- Domínguez AJ, Rodríguez VR, Honhold N. Epizootiología de los parásitos gastrointestinales en bovinos del estado de Yucatán. Vet Méx 1993; 24:189-93.

9.- Torres AF, Rodríguez VRI, Cámara SR. Efecto del parto sobre la eliminación de huevecillos de nemátodos y ooquistes de Eimeria en cabras criollas. Rev Biomed 1995; 6:208-15.

10.- Rodríguez VRI, Domínguez AJL, Torres AJF. Epidemiological factors associated to bovine coccidiosis in calves (Bos indicus) in a sub-humid tropical climate. Rev Biomed 1996; 7:211-8.

11.- Torres AJ. Nemátodos gastrointestinales en ovinos y Revista Biomédica caprinos. Actualización en enfermedades de ovinos y caprinos en el trópico. En Torres AJ, Ortega PA ed. Mérida: Universidad Autónoma de Yucatán; 1994. p. 61-7.

12.- Santamaría CN, Torres AJF, Rodríguez VRI. Efecto del peso al destete sobre el parasitismo gastrointestinal en cabritos en clima tropical. Rev Biomed 1995; 6:143-50.

13.- Anene BM, Nnaji TO, Chime AB. Intestinal parasitic infections of dogs in the Nsukka area of Enugu state, Nigeria. Prev Vet Med 1996; 17:89-94.

14.- Jenkins D. Intestinal parasites in dogs from an aboriginal community in New South, Wales. Australian Vet J 1993; 70:115-6.

15.- Quiñonez AF, Espaine AL, Rodríguez-Vivas RI, Domínguez-Alpizar JL. Contribución al estudio de los helmintos del tracto digestivo de perros de la ciudad de Mérida, Yucatán, México. Revista de la AMMVEPE 1998; 6:191-3.

16.- Bowman DD. Georgis' Parasitology for veterinarians. 6a ed. Philadelphia: Saunders Company; 1995. p. 145-6.

17.- Ezeokoli CD. Prevalence of gastrointestinal parasites in pet dogs in Zaria, Nigeria. Nigerian Vet J 1984; 13:557.

18.- Umoh JU, Asake TT. Prevalence of parasites and cysts in dogs faeces deposited on streets of staff quarters of Ahmadu Bello University, Zaria, Nigeria. Nigerian Vet J 1982; 11: 2.

19.- Levine N. Tratado de Parasitología Veterinaria. España: Acribia; 1978. p.6-10.

20.- Rodríguez-Vivas RI, Bolio GME, Domínguez-Alpizar JL, Aguilar FJA, Cob-Galera LA. Prevalencia de Dipylidium caninum en perros callejeros de la ciudad de Mérida, Yucatán, México. Rev Biomed 1996; 7:205-10.

21.- Boreham RE, Boreham PFL. Dipylidium caninum: Life cycle, epizootiology and control. Comp Cont Ed Prac Vet 1990; 5:667-75.

22.- Ferraris S, Reverso E, Parravicini LP. Dipylidium caninum in an infant. Europ J Pediatr 1993; 8:152.

23.- Watanabe T, Horii Y, Nawa Y. Case of Dipylidium caninum infection in an infant, the first case found in Miyazaki Prefecture, Japan. Jpn J Parasitol 1993; 3:2346. 
Parásitos gastrointestinales en animales domésticos.

24.- Rodríguez BJ, Allaway CE, Wassink GJ, Segura CJ, Rivera OT. Estudio de la avicultura de traspatio en el municipio de Dzununcán, Yucatán. Vet Méx 1996; $27: 215$ 9.

25.- Muirhead MR. Pig housing ang enviroment. Vet Record 1983; 1:13.

26.- Thornton K. Outdor pig production. London: Farming Press Limited; 1988. p. 3-4.

27.- Mackinnon J. Hut desing could damage pigs heath. Outdoor Production. London: a pig farming reference guide. 1991:33.

28.- Cordero CM, Hidalgo AM, Díez BN. Eimeriosis e Isosporosis. En: Cordero CM, Rojo VF ed. Parasitología Veterinaria. Madrid: McGraw Hill Interamericana; 1999. p.452-6.

29.- Vado SI. Monitoreo de indicadores de salud y producción en marranas gestantes bajo pastoreo en tres granjas del estado de Yucatán. Tesis de Maestría. Facultad de Medicina Veterinaria y Zootecnia. Universidad Autónoma de Yucatán. 1995. p. 90.

30.- Muller KD. Epidemiology, pathogenesis and control of major swine helminth parasites. Vet Clin NA 1986; 2:439-54.

31.- Tarazona VJ. Estrongilidosis causadas por grandes y pequeños estrongilos. En: Cordero CM, Rojo VF. Parasitología Veterinaria. Madrid: McGraw Hill Interamericana; 1999. p.545-60.

32.- Alcázar AA. Frecuencia de grandes strongilidos en ciego de caballos sacrificados en el rastro de Iztapalapa, D.F. Vet Méx 1989; 20:447.

33.- Pinzón NM. Estudio de las helmintiasis gastrointestinales en equinos de la escuela militar de especialistas en equitación. Tesis de licenciatura. Facultad de Medicina Veterinaria y Zootecnia. Universidad Nacional Autónoma de México. 1977:23. 